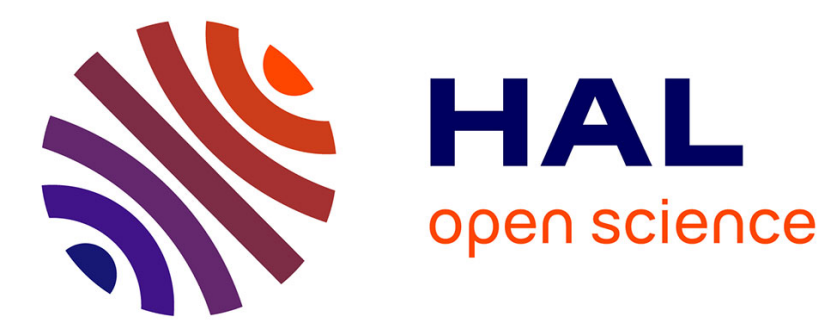

\title{
Influence de la présentation au verrat sur l'âge à la puberté des truies
}

Armelle Prunier

\section{To cite this version:}

Armelle Prunier. Influence de la présentation au verrat sur l'âge à la puberté des truies. Productions Animales, 1989, 2 (1), pp.65-72. hal-00895855

\section{HAL Id: hal-00895855 https://hal.science/hal-00895855}

Submitted on 1 Jan 1989

HAL is a multi-disciplinary open access archive for the deposit and dissemination of scientific research documents, whether they are published or not. The documents may come from teaching and research institutions in France or abroad, or from public or private research centers.
L'archive ouverte pluridisciplinaire HAL, est destinée au dépôt et à la diffusion de documents scientifiques de niveau recherche, publiés ou non, émanant des établissements d'enseignement et de recherche français ou étrangers, des laboratoires publics ou privés. 
INRA Prod. Anim., 1989, 2 (1), 65-72

\section{Armelle PRUNIER}

INRA Station de Recherches porcines Saint-Gilles

35590 L'Hermitage
Influence de la présentation au verrat sur l'âge à la puberté des truies

La mise en présence de jeunes truies avec un verrat permet d'avancer leur âge à la puberté de quelques jours à plusieurs semaines. Ce phénomène, connu sous le nom d'« effet mâle », est utilisé couramment par l'éleveur, de façon consciente ou non. En effet, lorsque celui-ci désire mettre à la reproduction ses jeunes truies, il les met en présence du verrat afin de détecter l'apparition de l'oestrus. De nombreuses expériences ont permis de définir les conditions d'efficacité de cet effet mâle alors que les mécanismes mis en jeu restent mal compris.

Chez la truie, la puberté est définie par l'apparition du premier oestrus qui coïncide le plus souvent avec la première ovulation et l'aptitude à mener une gestation à son terme. Une puberté précoce permet d'avancer l'âge à la mise à la reproduction des truies et d'améliorer leur productivité numérique par année de présence (Noguéra et Guéblez 1984). De plus, cela facilite leur insertion dans les bandes de truies sevrées puisqu'il est maintenant possible de contrôler le jour de venue en oestrus des femelles pubères par l'utilisation d'un progestagène de synthèse (Martinat-Botté et al 1977).

\footnotetext{
Résumé

Dans cette synthèse bibliographique nous avons successivement étudié la nature des signaux sensoriels mis en jeu lors de la mise en présence des truies et du verrat, la réaction de la femelle au plan endocrinien et les facteurs de variation de l'efficacité du mâle pour accélérer la venue en puberté des femelles.

Les signaux sensoriels mis en jeu sont multiples et impliquent probablement les différentes voies, olfactive, auditive, visuelle et tactile. La truie réagit à la présentation du verrat par une sécrétion accrue de cortisol, susceptible de modifier la sécrétion des hormones gonadotropes (LH et FSH). La diminution de l'âge à la puberté grâce à la mise en présence du mâle s'observe quel que soit le type génétique de la truie. Pour que l'effet soit maximal, il faut que le verrat soit mature et que les contacts physiques avec les femelles soient complets. Leur durée peut être limitée à quelques dizaines de minutes par jour. L'existence de contacts avec le mâle pendant le jeune âge (engraissement) n'altère pas l'efficacité de la mise en présence du verrat pendant la période prépubertaire.
}

L'âge moyen à la puberté varie très fortement d'un troupeau de truies à l'autre, de 5 à 9 mois suivant les auteurs. En plus des facteurs génétiques, de nombreux facteurs de l'environnement sont susceptibles de stimuler ou de retarder le développement sexuel. Ce sont notamment la claustration, la durée d'éclairement, la température, le stress et l'existence ou non de contacts avec d'autres congénères femelles ou mâles. L'influence stimulante des contacts avec le verrat, mise en évidence par Brooks et Cole en 1970 sur des truies croisées, a depuis lors fait l'objet de nombreuses études.

Cet article fait la synthèse des résultats bibliographiques concernant l'influence de la présentation au verrat sur la précocité sexuelle des truies. Dans un premier temps, nous analyserons la nature des signaux sensoriels mis en jeu et les conséquences au plan physiologique. Puis, nous rechercherons quels sont les facteurs susceptibles de moduler l'efficacité de la présentation au verrat.

Dans la plupart des études, il existe un groupe "témoin » de truies pour lesquelles la détection de l'oestrus doit se faire en l'absence du verrat. Cette recherche étant peu efficace dans cette condition (Signoret 1972), nous utiliserons comme critère de puberté l'âge à la première ovulation chaque fois que cela sera possible, celle-ci étant contrôlée par dosage de la progestérone ou par mise en évidence des corps jaunes après endoscopie ou abattage. 


\section{1 / Nature des signaux sensoriels mis en jeu}

Lors de la mise en présence de la truie avec le verrat, les signaux émis par celui-ci sont à la fois de nature olfactive, visuelle, auditive et tactile. Chaque composante joue un rôle actif pour permettre la manifestation du comportement d'oestrus de la femelle adulte (Signoret 1972). Aussi, on peut supposer que chacune intervienne pour stimuler l'apparition de la puberté. Nous avons donc recherché successivement le rôle joué par chaque type de signal et l'exis tence d'éventuels phénomènes d'interaction ou de synergie.

\section{1 / Signaux olfactifs}

La nécessité de la présence des signaux olfactifs a été mise en évidence par Kirkwood et al (1981 et 1983). Dans la première expérience, ces auteurs ont montré que des truies, rendues anosmisques par ablation des bulbes olfactifs et mises en présence d'un verrat quotidiennement, ont un âge à la puberté identique à celui de femelles non opérées isolées du mâle, et retardé par rapport à des truies, non opérées ou opérées « à blanc » et subissant l' « effet mâle». Dans la seconde expérience, l'utilisation d'un mâle castré traité aux androgènes, ayant un comportement sexuel normal mais dont la sécrétion de stéroïdes odorants est supprimée (Booth 1980), s'est avérée beaucoup moins efficace pour stimuler l'apparition de la puberté que celle d'un verrat entier.

Malheureusement, les expériences visant à identifier les signaux olfactifs mis en jeu se sont révélées infructueuses. Aucun des principaux stéroïdes odorants contenus dans la salive et l'urine de verrat entier ne permet d'avancer l'âge à la puberté des jeunes truies (tableau 1).

Par ailleurs, si les androstènes jouaient un rôle important, on peut supposer que des verrats ayant un très fort potentiel de sécrétion seraient plus stimulants pour les truies. Cette hypothèse a été testée par Booth (1987) en comparant l'influence de l'introduction d'un verrat Large White à celle d'un mâle miniature de race Göttingen, dont la concentration d'androsténone de la salive est 10 à 20 fois plus forte. L'âge à la puberté des femelles a été similaire avec les verrats des deux races. Il ne semble donc pas que le potentiel de sécrétion des androstènes soit un facteur important.

Pearce et Hughes (1987a) ont également essayé d'identifier la nature des signaux olfactifs qui jouent un rôle actif. Pour cela, ils ont utilisé un mâle castré traité aux androgènes portant ou non autour du cou une gaze imbibée de différents produits odorants. L'utilisation d'urine de mâle entier, renforcée ou non par une solution des deux androstènes, n'a pas eu d'effet significatif sur la venue en puberté des femelles (tableau 2). Cependant, la diffusion simultanée d'un enregistrement du " chant de cour" (grognements émis par un verrat en présence d'une truie en oestrus) permet d'obtenir une précocité sexuelle proche de celle observée avec un verrat entier.

Il semble donc que les signaux olfactifs pris isolément soient insuffisants mais agissent en synergie avec d'autres stimuli pour avancer l'âge à la puberté des truies.

\section{2 / Signaux tactiles}

La présence du verrat dans une loge séparée de celles des truies par des barreaux permet des contacts olfactifs, auditifs et visuels complets alors que les contacts physiques sont très réduits. La précocité sexuelle des truies est alors améliorée mais avec une efficacité moindre que dans le cas où le verrat pénètre dans la loge des femelles (tableau 3). L'utilisation d'un mâle castré traité aux androgènes permet d'obtenir des contacts physiques normaux, le comportement sexuel mâle étant maintenu. Dans ce cas, la venue en puberté des femelles est retardée d'environ 1 mois par rapport à celle observée avec un mâle entier (tableau 2). Les signaux tactiles jouent donc un rôle actif dans l'« effet verrat » mais sont insuffisants.

\section{3 / Signaux visuels et auditifs}

Dans le cas où ces deux types de signaux sont maintenus par la présence d'un mâle

Tableau 1. Influence de divers composés odorants sécrétés par le verrat sur le pourcentage de truies ayant ovulé à un âge donné.

\begin{tabular}{|l|c|c|}
\hline Référence & Kirkwood et al 1983 & Booth 1984 \\
\hline Pas de traitement & $25 \mathrm{ab}$ & $17 \mathrm{a}$ \\
Extraits de glandes salivaires & $12 \mathrm{a}$ & $\mathrm{nd}$ \\
$5 \alpha$-androstémone & $19 \mathrm{ab}$ & $\mathrm{nd}$ \\
$3 \alpha$-androsténol & $44 \mathrm{~b}$ & $\mathrm{nd}$ \\
$5 \alpha$-androsténone $+3 \alpha$-androsténol & $31 \mathrm{ab}$ & $\mathrm{nd}$ \\
$5 \alpha$-androsténone $+3 \alpha$-androsténol + salive & $\mathrm{nd}$ & $33 \mathrm{a}$ \\
Urine & $20 \mathrm{ab}$ & $\mathrm{nd}$ \\
Verrat & $100 \mathrm{c}$ & $100 \mathrm{~b}$ \\
\hline
\end{tabular}

a, b, c : pour une même expérience, les pourcentages suivis d'une lettre différente sont significativement différent à $\mathrm{P}<0,05$.

nd : non déterminé 
Tableau 2. Influence des signaux olfactifs et auditifs émis par le verrat sur l'âge à la puberté (jours) de truies croisées (d'après Pearce et Hughes 1987 a).

\begin{tabular}{|l|c|c|}
\hline & Essai 1 & Essai 2 \\
\hline Mâle entier & $197 \mathrm{a}$ & $197 \mathrm{a}$ \\
\hline Mâle castré traité aux androgènes & & \\
$\quad$ sans rien & $232 \mathrm{~b}$ & nd \\
+ urine & $231 \mathrm{~b}$ & nd \\
+ urine + androstènes & $222 \mathrm{~b}$ & $229 \mathrm{~b}$ \\
+ chant de cour & nd & $213 \mathrm{~b}$ \\
+ urine + chant de cour & nd & $209 \mathrm{ab}$ \\
\hline
\end{tabular}

a, b : pour un même essai, les moyennes suivies d'une lettre différente diffèrent significativement. nd : non déterminé.

Tableau 3. Influence de la limitation des contacts physiques entre le verrat et les truies sur leur venue en puberté.

\begin{tabular}{|c|c|c|c|c|}
\hline $\begin{array}{l}\text { Mâle dans loge adjacente } \\
\text { Mâle dans la loge des truies }\end{array}$ & - & $\begin{array}{l}+ \\
-\end{array}$ & $\overline{+}+$ & $\begin{array}{l}+ \\
+\end{array}$ \\
\hline $\begin{array}{l}\text { Age au } 1^{\text {er }} \text { oestrus (jours) } \\
\text { (d'après Karlbom 1982) }\end{array}$ & $198 \mathrm{a}$ & $183 \mathrm{~b}$ & nd & $163 \mathrm{C}$ \\
\hline $\begin{array}{l}\text { Age à la } 1^{\text {re }} \text { ovulation (jours) } \\
\text { (d'après Walton 1985) }\end{array}$ & 197 & 189 & nd & nd \\
\hline $\begin{array}{l}\text { \% de truies ayant ovulé à } 210 \text { jours } \\
\text { (d'après Deligeorgis et al 1984) }\end{array}$ & $19 a$ & $38 a$ & $76 \mathrm{~b}$ & nd \\
\hline $\begin{array}{l}\% \text { de truies ayant ovulé à } 180 \text { jours } \\
\text { (d'après Walton } 1984 \text { ) }\end{array}$ & 0a & $83 \mathrm{~b}$ & nd & nd \\
\hline
\end{tabular}

a, b, c : pour une même expérience, les moyennes ou les pourcentages suivis d'une lettre différente diffèrent significativement à $\mathrm{P}<0,05$.

nd : non déterminé.

entier dans la loge adjacente, la puberté est peu ou pas avancée (tableau 3). Lorsqu'un mâle castré non supplémenté en stéroïdes est utilisé, les signaux visuels sont quasi-normaux, la morphologie étant peu modifiée, alors que les signaux olfactifs, auditifs et tactiles sont profondément altérés. Dans ce cas, la précocité sexuelle est similaire à celle de truies isolées de tout verrat (Alexander et Froseth 1981). Enfin, la diffusion d'un enregistrement du " chant de cour " en même temps que la mise en présence d'un mâle castré traité aux androgènes ne permet pas d'améliorer la précocité sexuelle des truies (tableau 2). Les signaux visuels et auditifs sont donc insuffisants. Cependant, les signaux auditifs semblent jouer un rôle actif puisque dans l'expérience de Pearce et Hughes (1987a), l'influence du mâle castré devient similaire à celle de l'entier seulement lorsque ces signaux sont introduits en plus des trois autres types (tableau 2).

Pour avancer l'âge à la puberté des truies, les quatre types de signaux émis par le verrat sont donc insuffisants lorsqu'ils sont isolés les uns des autres mais ils semblent tous jouer un rôle actif. Il y a donc complémentarité entre les différents signaux sensoriels et probablement nécessité pour devenir efficaces qu'ils soient tous réunis.

\section{2 / Conséquences physiologiques de l'introduction du verrat}

Le développement sexuel de la truie se caractérise par l'existence d'une phase d'activation, vers l'âge de 3 mois, durant laquelle les ovaires se développent rapidement grâce aux sécrétions élevées d'hormones gonadotropes, LH et FSH (Camous et al 1985, Prunier 1985, figure 1). Puis, à partir de 4-5 mois d'âge, les ovaires atteignent leur développement prépubertaire maximal et les sécrétions gonadotropes se stabilisent à un faible niveau. C'est la phase d'attente au cours de laquelle la puberté est susceptible d'apparaître à n'importe quel moment sous l'action des stimuli de l'environnement. Au cours de cette phase, le rétrocontrôle positif, c'est-à-dire la possibilité de stimuler la sécrétion de LH par élévation du niveau plasmatique d'oestradiol, est fonctionnel sans que l'on sache s'il est complètement mature. Par ailleurs, Fonda et al (1984) et Pearce et al (1988) ont montré qu'à ce stade, les corticostéroïdes surrénaliens sont susceptibles de modifier la sécrétion de LH.

Le verrat, qui est généralement introduit lorsque les femelles ont 140-180 jours, agit donc le plus souvent sur la durée de la phase d'attente.
Chaque signal sensoriel émis par le verrat joue un rôle actif dans l'effet mâle mais aucun n'est efficace à lui seul. 
Figure 1.Caractéristiques physiologiques du développement sexuel de la truie (d'après Prunier 1985).

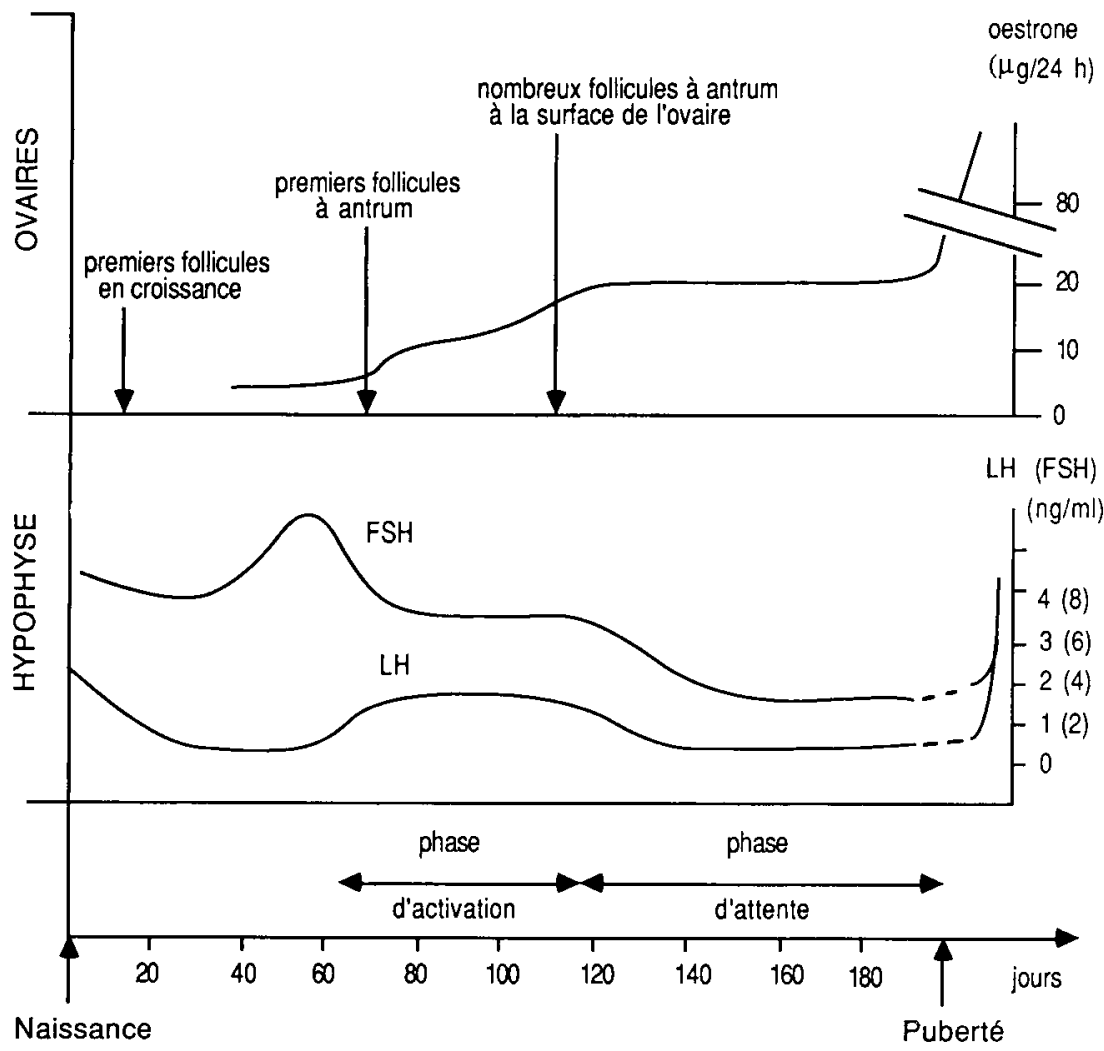

Figure 2.Influence de la mise en présence d'un mâle entier ou castré traité aux androgènes sur la concentration plasmatique de cortisol chez la truie (d'après Pearce et Hughes 1987).

La présentation au verrat provoque un stress, dès lors que les animaux se retrouvent dans une même loge ; mais ce stress n'est pas suffisant pour induire une puberté précoce.

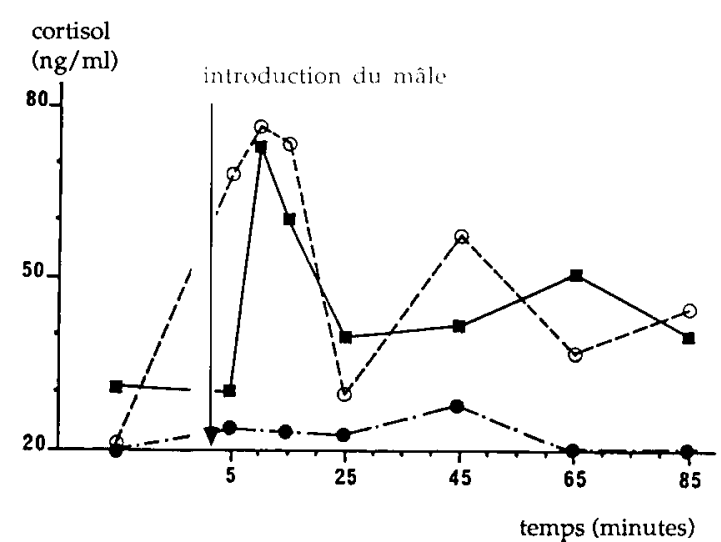

mâle castré traité aux androgènes

mâle dans la loge adjacente
La réduction de celle-ci suppose qu'il y a activation de l'axe hypothalamus-hypophyseovaires. Le problème est de savoir où se situe la stimulation exercée par le verrat et quels sont les mécanismes mis en jeu. Malheureusement, chez le porc, peu d'études ont été réalisées dans ce domaine et seules quelques questions limitées ont obtenu une réponse.

\section{1 / Maturation du rétrocontrôle positif}

Hughes et Cole (1978) ont recherché si l'introduction du verrat pouvait accélérer la maturation du rétrocontrôle positif des oestrogènes sur la sécrétion de LH. Des truies traitées au benzoate d'oestradiol (oestrogène de synthèse ayant une demi-vie de longue durée) ont été mises en présence ou non d'un verrat. La réponse a été similaire dans les deux groupes: 6 femelles sur 10 et 6 femelles sur 9 , respectivement pour les lots avec et sans mâle ont eu un oestrus accompagné de l'ovulation, dans les $\mathbf{5}$ jours suivant l'injection. Il semble donc que la maturation du rétrocontrôle permettant l'induction d'un pic ovulatoire de LH n'a pas été modifiée par la présence du mâle.

\section{2 / Sécrétion de LH}

Signoret et al (communication personnelle) n'ont pas observé, chez la truie, une augmentation immédiate de la sécrétion de LH lors de la mise en présence du mâle comme cela avait été montré chez la brebis (Poindron et al 1980).

\section{3 / Axe corticotrope}

Enfin, Pearce et Hughes (1987b) ont recherché s'il y avait activation de l'axe corticotrope lors de la présentation des truies au verrat. Il y a effectivement augmentation de la sécrétion de cortisol mais seulement si les animaux ont un contact physique complet (figure 2). Cependant, cette réaction n'est pas suffisante pour expliquer l' «effet mâle» puisqu'on la retrouve avec un mâle castré supplémenté en androgènes alors que ce type d'animal ne permet pas d'avancer l'âge à la puberté (cf 1.1).

\section{3 / Facteurs de variation}

\section{1 / Génotype}

L’âge à la puberté des truies varie entre les races et les animaux croisés ont une puberté plus précoce que ceux de race pure. On peut se demander si ces variations sont liées à une différence de sensibilité des femelles aux stimuli de l'environnement, et notamment à la mise en présence du verrat. La majorité des études a été réalisée chez des femelles croisées et montre que l'introduction du verrat avance l'âge à la puberté (tableau 4). Les expériences faites chez des truies de race pure Large White, pour laquelle la puberté est relativement tardive, révèlent également un effet positif du mâle (tableau 4). La mise en présence du mâle est donc bénéfique quel que soit le type génétique, sans que l'on puisse dire si un type est plus 
Tableau 4. Influence de la mise en présence d'un verrat sur l'âge à la puberté (jours) ou le pourcentage de femelles pubères à la fin de l'expérience.

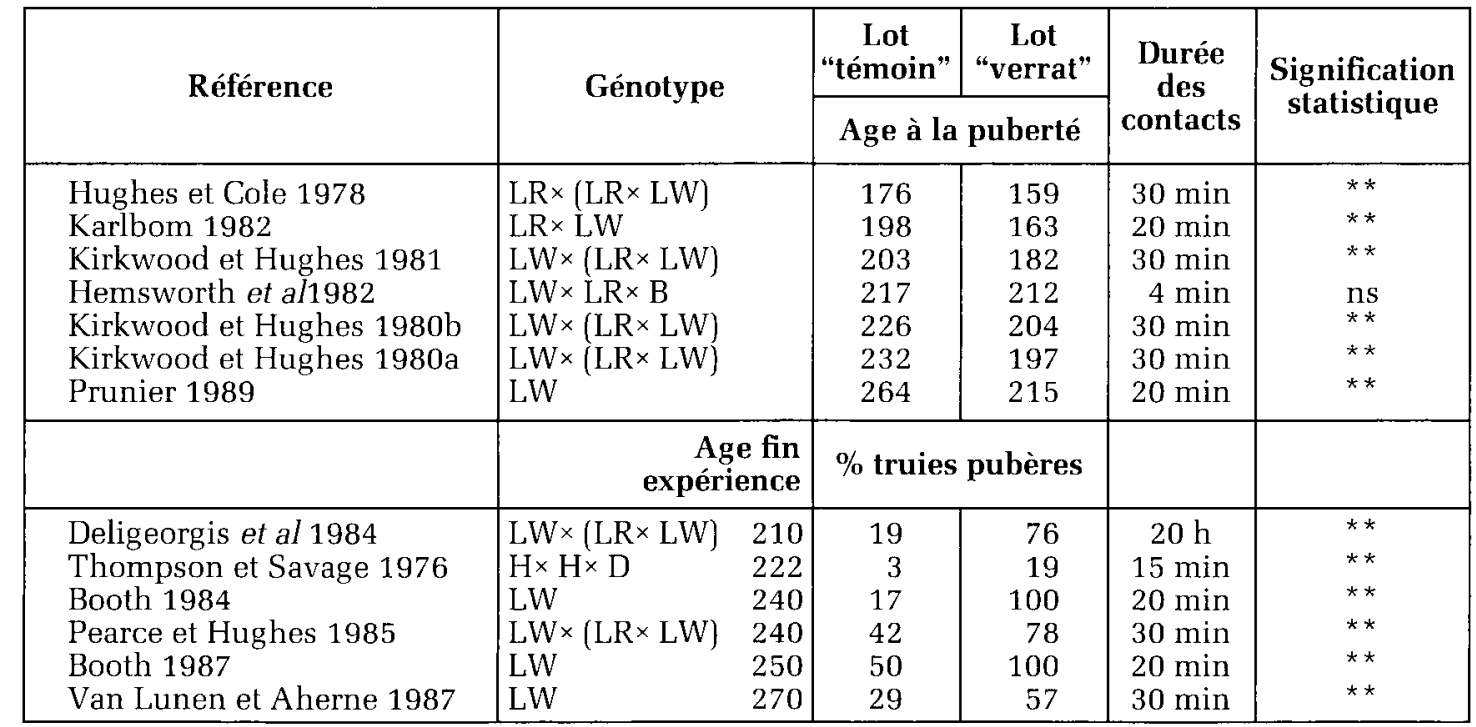

Influence significative $\left({ }^{*}\right)$ ou non (ns) du verrat

LR : Landrace, LW : Large White, B : Berkshire, D : Duroc, H : Hampshire,

sensible que l'autre en raison de l'absence d'expérience dans ce domaine. Il faut remarquer que, dans ces diverses expériences, le verrat et les truies sont généralement du même génotype. Cependant, lorsque le verrat est d'une race différente, la réponse des truies ne semble pas altérée (Booth 1987).

\section{2 / Age des animaux}

\section{a / Verrat}

Le comportement sexuel et la sécrétion de stéroïdes du verrat dépendent en premier lieu du stade de maturité sexuelle auquel il se trouve. Aussi, l'âge du mâle conditionne vraisemblablement son efficacité pour stimuler l'apparition de la puberté chez les femelles. En effet, un verrat croisé, âgé de 6 mois et demi, est sans effet sur l'âge au premier oestrus des truies mises en sa présence alors qu'à 11 mois, il est aussi efficace qu'un adulte (figure 3).

\section{b / Truies}

Afin d'optimiser le moment de l'introduction $\mathrm{du}$ verrat dans un troupeau de truies, il est nécessaire de savoir s'il existe un stade à partir duquel les jeunes femelles deviennent réceptives à la présence du verrat et si cette sensiblité varie au cours de leur développement sexuel. Ce problème a été posé par plusieurs auteurs avec deux approches différentes. Certains ont comparé les âges à la puberté de truies mises en présence d'un verrat à différents moments au cours de la période prépubertaire (au delà de 125 jours). D'autres ont recherché si la présence de mâles contemporains ou adultes pendant la phase d'engraissement (70 à 140-160 jours) influençait l'efficacité des contacts avec le mâle pendant la période prépubertaire.
Les travaux de Hughes et Cole (1976) suivis de ceux de Kirkwood et Hughes (1979) et de Eastham et al (1986a) montrent que le délai entre l'introduction du verrat et le premier oestrus détecté diminue rapidement entre 125 et 140 jours d'âge (-2 jours de délai lorsque l'âge des truies augmente de 1 jour), alors qu'il varie peu ultérieurement $(-0,3$ jour $)$. L’âge à la puberté diminue donc avec l'âge à l'introduc-

Figure 3. Influence de l'âge du verrat sur l'âge moyen à la puberté des truies mises en présence de celui-ci 30 minutes par jour (d'après Kirkwood et Hughes 1981).

$a-b$ : l'écart est signicatif à $P<0,05$

Age à la première ovulation (jours)

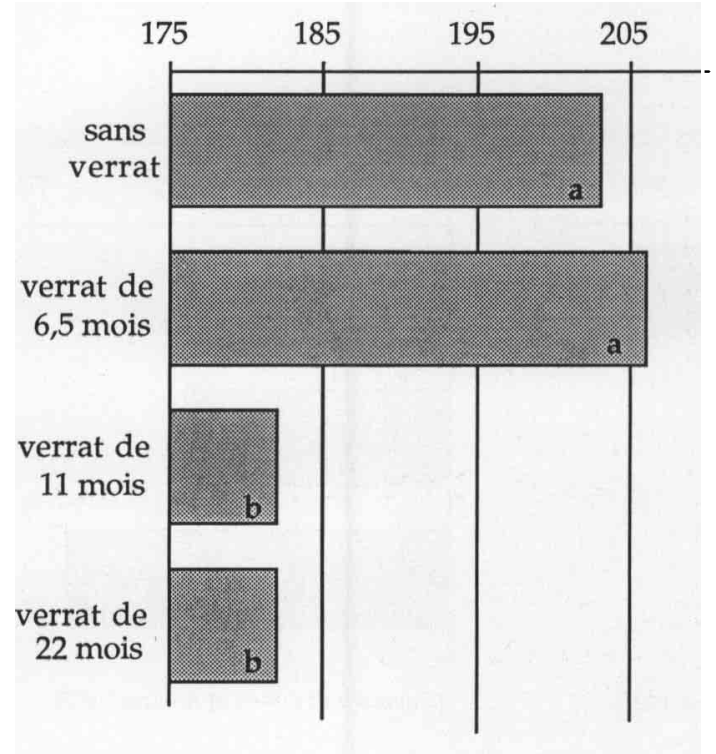


Tableau 5. Influence de l'existence des contacts avec le verrat pendant la phase d'engraissement sur lâge à la puberté (jours) des truies.

\begin{tabular}{|c|c|c|c|c|}
\hline $\begin{array}{l}\text { de } 70 \text { à } 160 \text { jours } \\
\text { de } 160 \text { à la puberté }\end{array}$ & $\begin{array}{l}- \\
-\end{array}$ & $\begin{array}{c}\text { ntacts } \\
+ \\
-\end{array}$ & $\begin{array}{c}\text { âle(s) e } \\
- \\
+\end{array}$ & $\begin{array}{l}+ \\
+\end{array}$ \\
\hline Paterson et al 1980 & $220 \mathrm{a}$ & $197 \mathrm{~b}$ & $182 \mathrm{C}$ & $181 \mathrm{C}$ \\
\hline Eastham et al 1984 & nd & nd & 168 & 167 \\
\hline Eastham et al $1986 \mathrm{~b}$ & nd & nd & 181 & 184 \\
\hline Eastham et Cole 1987 & nd & nd & 176 & 185 \\
\hline
\end{tabular}

a, b, c : pour une même expérience, les moyennes suivies d'une lettre différente diffèrent significativement, $\mathrm{P}<0,05$. nd : non déterminé.

\section{Les contacts précoces avec le verrat ne diminuent pas l'efficacité des contacts pendant la phase prépubertaire.}

Figure 4. Influence de la durée des contacts avec le verrat sur la venue de la puberté des truies. $a-b$ : l'écart est significatif à $P<0,05$. tion du verrat jusqu'à 140 jours puis la relation s'inverse et l'âge à la puberté augmente. Cependant, cette conclusion est très criticable car elle repose sur de faibles effectifs par lot ( $n=4$ à 10). De plus, aucun contrôle de l'oestrus ou de l'ovulation, permettant de déterminer si certaines femelles étaient déjà pubères au moment de l'introduction du verrat, n'a été effectué alors que cette hypothèse est probable pour les truies les plus âgées.

Les études présentées au tableau 5 montrent que s'il y a eu contact avec des mâles contemporains (Paterson et al 1980) ou adultes (Eastham et al 1984 et 1986b, Eastham et Cole 1987) pendant la phase d'engraissement, la présentation du verrat pendant la période prépubertaire reste efficace. On constate même que les contacts précoces ont un effet favorable sur l'âge à la puberté de femelles maintenues à l'écart du mâle pendant la période prépubertaire.

Il est donc souhaitable pour obtenir une puberté précoce des truies de les mettre en contact avec le verrat le plus tôt possible.

\section{3 / Qualité des contacts}

\section{a / Durée des contacts}

Les contacts entre les truies et le verrat sont souvent limités à la détection des chaleurs et sont donc relativement courts, de 5 à 30

minutes par jour en général. Cela suffit à avancer l'apparition de la puberté (tableau 4) et semble même aussi efficace qu'une présence continue du mâle parmi les femelles (figure 4).

\section{b / Nombre de verrats}

Brooks et Cole (1970) ont supposé qu'en alternant différents verrats d'un jour à l'autre, il serait possible d'augmenter la stimulation. En effet, cette technique permettrait d'éviter l'accoutumance éventuelle des femelles à un mâle particulier et de pallier la déficience possible de l'un d'eux. La puberté est effectivement plus précoce avec plusieurs verrats utilisés alternativement qu'avec un seul : respectivement 100 et $45 \%$ de truies ont eu un oestrus avant 185 jours d'âge. Malheureusement, cet effet mis en évidence sur de faibles effectifs de femelles (n=12 par lot) n'a pas fait l'objet d'étude ultérieure qui permettrait de le valider. Par ailleurs, la présentation des femelles à plusieurs mâles simultanément n'améliore pas et a même tendance à diminuer l'efficacité de l'« effet mâle » : le pourcentage de femelles ayant ovulé à 270 jours a été de $68 \%$ avec un seul verrat et de $48 \%$ avec 4 verrats $(n=21$ par lot, Van Lunen et Aherne 1987).

\section{c / Mode de logement des truies}

Les contacts entre truies et verrat ont lieu dans la loge du verrat ou dans celle des truies. Dans le premier cas, l' « effet mâle » s'accompagne d'un stimulus dû au changement de loge (cf 2.4). Dans le second cas, le fait que les truies soient en loges collectives ou à l'attache est susceptible de modifier la qualité des interactions entre le verrat et les truies. Lorsque le mâle pénètre dans une loge collective de truies, on constate un effet positif sur l'âge à la puberté qui est très marqué et répétable tandis que si les truies sont attachées, l'effet est variable (tableau 6). Cette dernière situation, peu fréquente chez les éleveurs, montre donc l'existence d'une interaction entre le mode de logement des truies et l' " effet verrat". Aussi, on peut se demander si pour des truies élevées en loge individuelle ou en loge collective avec une forte densité d'animaux, l'« effet du verrat » n'est pas altéré.

\section{4 / Présence d'autres stimuli}

Chez de nombreux éleveurs de porcs et dans la plupart des études expérimentales, la mise 
Tableau 6. Variations de «l'effet mâle"sur l'âge à la $1^{r e}$ ovulation (jours) des truies en fonction de leur mode de logement (d'après Prunier 1989).

\begin{tabular}{|c|cc|cc|}
\hline & \multicolumn{2}{|c|}{ Loges collectives } & \multicolumn{2}{c|}{ Attache } \\
Passage du verrat & non & 2 fois/jour & non & 2 fois/jour \\
\hline Essai 1 & $>300$ a.u & $209 \mathrm{C}$ & $297 \mathrm{~b}$ & 294 b.u \\
Essai 2 & 246 a.v. & $217 \mathrm{~b}$ & $240 \mathrm{a}$ & $205 \mathrm{~b} . \mathrm{v}$ \\
Essai 3 & 258 a.v. & $220 \mathrm{~b}$ & $255 \mathrm{a}$ & 229 b.v. \\
\hline
\end{tabular}

a. b. c. : pour un même essai. les àges à la puberté suivis d'une lettre différente diffèrent significativement entre traitements.

u. v. : pour un même traitenent, les âges à la puberté suivis d’une lettre différente diffèrent significativement entre essais.

Tableau 7. Influence de lintroduction du verrat, couplée ou non au déplacement des truies, sur l'apparition de la puberté de celles-ci.

\begin{tabular}{|l|c|c|c|c|}
\hline $\begin{array}{l}\text { Contact avec le verrat } \\
\text { Déplacement quotidien des truies }\end{array}$ & $\begin{array}{l}- \\
-\end{array}$ & $\begin{array}{c}- \\
+\end{array}$ & $\begin{array}{c}+ \\
+\end{array}$ \\
\hline $\begin{array}{l}\text { \% de truies ayant ovulé à 270 jours } \\
\text { (d'après Van Lunen et Aherne 1987) }\end{array}$ & $29 \mathrm{~b}$ & nd & $48 \mathrm{ab}$ & $68 \mathrm{a}$ \\
\hline $\begin{array}{l}\text { Age à la 1"i ovulation (jours) } \\
\text { (d'après Pearce et Hughes 1985) }\end{array}$ & $245 \mathrm{~b}$ & $245 \mathrm{~b}$ & nd & $205 \mathrm{a}$ \\
\hline
\end{tabular}

en présence des truies et du verrat s'accompagne d'autres modifications de l'environnement également susceptibles de moduler l'âge à la puberté. Il est donc important de rechercher l'effet propre du verrat et, surtout, de déterminer si les différentes stimulations s'additionnent ou même agissent en synergie.

Le changement d'élevage est un cas de modification maximale de l'environnement des jeunes truies. Il permet d'induire la puberté dans un délai de 10 jours chez 26 à $72 \%$ de femelles croisées pesant environ $100 \mathrm{~kg}$ (Du Mesnil du Buisson et Signoret 1962, Martinat et al 1970, Martinat-Botté et al 1989). Les femelles sont alors soumises à un stress intense dû au transport en camion, à la vaccination, au lavage, au changement d'environnement physique et social... A ce "choc émotionnel » s'ajoute la mise en présence du verrat lors de la détection des chaleurs. Cependant, Martinat et al (1970) ont montré que l'apparition de la puberté dans les 10 jours qui suivent le change-

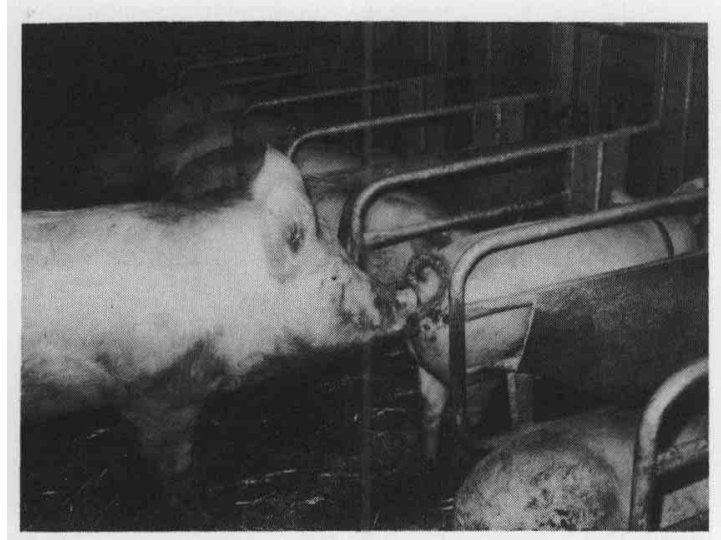

La présence du verrat a toujours un effet marqué lorsque les truies sont en loges collectives. Par contre cet effet est variable lorsqu'elles sont à l'attache. ment d'élevage était essentiellement dû au « choc émotionnel. »

Dans certaines expériences, l'introduction du verrat s'accompagne de celle d'une autre perturbation qui persistera pendant toute la durée de l'expérience, tel que le déplacement quotidien des truies vers la loge du verrat ou la réalisation de prises de sang à intervalles de temps réguliers. Malheureusement, très peu d'études ont recherché l'existence d'une interaction entre l'introduction du verrat et ces autres stimuli. Le déplacement quotidien des truies vers une autre loge, sans effet par lui-même, n'améliore pas significativement l'efficacité de l'introduction du verrat (tableau 7).

\section{Conclusion}

Cette synthèse des résultats bibliographiques permet de confirmer l'importance de la mise en présence des truies et du verrat pour avancer l'âge à la puberté des femelles. La nature des signaux sensoriels mis en jeu est certainement complexe. Aucun type de signal, olfactif, visuel, auditif ou tactile ne permet à lui seul d'agir sur la précocité sexuelle des femelles bien que chacun semble jouer un rôle actif. Aussi, il est vraisemblable que les différents signaux jouent un rôle complémentaire et qu'il est nécessaire qu'ils soient tous réunis pour devenir efficaces. Les mécanismes endocriniens n'ont pas encore été élucidés. En fait, les expériences réalisées dans ce domaine sont trop peu nombreuses et n'ont pas permis d'obtenir de résultats concluants.

En ce qui concerne les conditions d'efficacité du verrat, plusieurs points importants apparaissent. Pour obtenir une stimulation maximale du développement sexuel des truies, le verrat doit être adulte et ses contacts physiques avec la truie doivent être complets, ceci n'étant possible que si les animaux se retrouvent dans la même loge. Par contre, on peut se contenter d'une mise en présence de courte durée, de 
l'ordre de quelques dizaines de minutes par jour. Des contacts très précoces, pendant la phase d'engraissement, ne sont pas préjudiciables tandis que le maintien à l'attache des truies prépubères est défavorable. La présence d'autres stimuli ne semble pas négative mais l'existence d'un effet synergique avec celui de l'introduction du verrat n'a pas été démontrée.

\section{Remerciements}

L'auteur remercie Michel Etienne, Jean-Yves Dourmad Marie-Christine Meunier-Salaun (INRA Saint-Gilles) et Françoise Martinat-Botté (INRA Nouzilly) pour leur évaluation critique du manuscrit.

\section{Références bibliographiques}

BOOTH W.D., 1980. A study of some major testicular steroids in the pig in relation to their effect on the development of male characteristics in the prepubertally castrated boar. J. Reprod. Fert., 59, 155-162.

BOOTH W.D, 1984. A note on the significance of boar salivary pheromones to the male-effect on puberty attainment in gilts. Anim. Prod., 39, 149-152

BOOTH W.D. 1987. A note on the influence of a Göttingen miniature boar on puberty attainment in large White gilts. Anim. Prod., 44, 165-168.

BROOKS P.H., COLE D.J.A, 1970. The effect of the presence of a boar on the attainment of puberty in gilts. J Reprod. Fert., 23, 435-440.

CAMOUS S., PRUNIER A., PELLETIER J., 1985. Plasma prolactin, LH. FSH and estrogen excretion pattern in gilt during sexual development. J. Anim. Sci., 60, 1308-1317. DELIGEORGIS S.G., LUNNFY D.C., ENGLISI I P.R., 1984 A note on the efficacy of complete V. partial boar exposure on puberty attainment in the gilt. Anim. Prod., 391 145-147.

DU MESNIL DU BUISSON F., SIGNORET I.P., 1962 Influences des facteurs externes sur le déclenchement de la puberté chez la truie. Ann. Zootech., 11, 53-59.

EASTHAM P.R., DICK G.W., COLE D.J.A., 1984. Reproduction in the gilt 5 . The effect of the presence of the mature male during rearing on puberty attainment Anim. Prod., 39, 277-282

FASTHAM P.R., DICK G.W., COLE D.J.A., 1986a. The effect of age at stimulation by relocation and first mature boar contact on the attainment of puberty in the gilt Anim. Reprod. Sci., 12, 31-38.

EASTHAM P.R., DICK G.W., COLE D.J.A., 1986b. Reproduction in the gilt. 6 . The effect of various degrees of mature boar contact during rearing on puberty attainment. Anim. Prod., 43, 343-349.

EASTHAM P.R., COLE D.J.A., 1987. Reproduction in the gilt. Exposure of young gilts to the same mature boar and relocation: effects on the attainment of puberty. Anim. Prod., 44, 435-441.

FONDA E.S., RAMPACECK G.B., KRAELING R.R., 1984 The effect of adrenocorticotropin or hydrocortisone on serum luteinizing hormone concentrations after adrenalectomy and/or ovariectomy in the prepubertal gilt. Endocrinology, 114, 268-273.

HEMSWORTH P.H., CRONIN G.M., HANSEN C., 1982 The influence of social restriction during rearing on the sexual behavior of the gilt. Anim. Prod, 35, 35-40.

HUGHES P.E., COLE D.J.A., 1976. Reproduction in the gilt. 3. The influence of gilt age at boar introduction on the attainmant of puberty. Anim. Prod., 23, 89-94

HUGHES P.E., COLE D.J.A., 1978. Reproduction in the gilt. 3 . The effect of exogenous oestrogen on the attainment of puberty and subsequent reproductive performance. Anim. Prod., 27, 11-20.

KARLBOM I., 1982. Attainment of puberty in females pigs: Influence of boar stimulation. Anim. Reprod. Sci. 4, 313-319

KIRKWOOD R.N., HUGHES P.E., 1979. The influence of age at first boar contact on puberty attainment in the gilt Anim. Prod., 29, 231-238.
KIRKWOOD R.N., HUGHES P.E., 1980a. A note on the efficacy of continuous $V$. limited boar exposure on puberty attainment in the gilt. Anim. Prod., 31, 205-207.

KIRKWOOD R.N.. HUGHES P.E., 1980b. A note on the influence of "boar effect component stimulin on pub. erty attainment in the gilt. Anim. Prod., 31, 209-211.

KIRKWOOD R.N., HUGHES P.E., 1981. A note on the influence of boar age on its ability to advance puberty in the gilt. Anim. Prod.. 32, 211-213.

KIRKWOOD R.N., FORBES J.M., HUGHES P.E., 1981. Influence of boar contact on attainment of puberty in gilts after removal of the olfactory bulbs. J Reprod. Fert., 61, 193-196.

KIRKWOOD R.N., HUGHES P.E., 1983. The influence of boar-related odours on puberty attaiment in gilts. Anim. Prod., 36, 131- 136.

MARTINAT F.. LEGAULT C., DU MESNIL DU BUISSON F., OLI.IVIER L., SIGNORET J.P., 1970. Etude des retards de puberté chez la truie. Journées de la Rech. Porcine en France, 2, 47-57

MARTINAT-BOTTE F, BARITEAU F, FORGERIT Y MACAR C., MOREAU A., TEROUI M., SIGNORET I.P. 1989. Introduction des cochettes dans l'élevage en bandes. I. Déclenchement. Journées de la Rech. Porcine en France, 21 (sous presse).

MARTINAT-BOTTE F., MAULEON P., GAUTIER J., 1977 Maitrise des cycles sexuels des truies nullipares. Comparaison de deux progestagènes : Ia northandronolone et le RU 2267. Journées de la Rech. Porcine en France, 9, 23 27.

NOGUERA J.L., GUEBLEZ R., 1984. Incidence de l'âge à la première mise bas et de la taille de la première portée sur la carrière de la truie. Journées de la Rech. Porcine en France, 16, 135-144.

PATERSON A.M., I.INDSAY D.R., 1980. Induction of puberty. 1. The effects of rearing conditions on reproductive performance and response to mature boars after early puberty. Anim. Prod, 31, 291-297.

PEARCE G.P., HUGHES P.E., 1985. The influence of daily movement of gilts and the environment in which boar exposure occurs on the efficacy of boar related precocious puberty in the gilt. Anim. Prod., 40, 161-167.

PEARCE G.P., HUGHES P.E. 1987a. The influence of boar- component stimuli on puberty attainment in the gilt. Anim. Prod., 44, 293-402.

PEARCE G.P. HUGHES PE, 1987b. The influence of male contact on plasma cortisol concentrations in the prepubertal gilt. J. Reprod. Fert., 80, 417424.

PEARCE G.P., PATERSON A.M., HUGHES PE, 1988. Effect of short-term elevations in plasma cortisol concentration on LH secretion in prepubertal gilts. I. Reprod. Fert., 83, 413-418.

POINDRON P., COGNIE Y., GAYERIE F., ORGEUR P., OLDHAM C.M., RAVAULT J.P., 1980. Changes in gonadotrophin and prolactin levels in isolated (seasonally or lactationally) anovular ewes associated with ovulation caused by the introduction of the rams. Physiol. Behav., $25,227-236$.

PRUNIER A., 1985. Physiologie du développement sexuel de la jeune truie. Journées de la Rech. Porcine en France, 17. $123-138$

PRUNIER A., 1989. Influence de l'introduction du verrat sur l'âge à la puberté de truies élevées en groupes ou à l'attache. Journées de la Rech. Porcine en France, 21 (sous presse)

SIGNORET J.P., 1972. Contribution à l'étude des mécanismes éthologiques et endocriniens du comportement sexuel de la truie (Sus scrofa L.). Thèse d'Etat. Université Paris VI.

THOMPSON L.H, SAVAGE J.S., 1978. Age at puberty and ovulation rate in gilts in confinement as influenced by exposure to boar. J. Anim. Sci., 47, 1141-1144.

VAN LUNEN T.A., AHERNE F.X., 1987. Influence of method of boar exposure on age at puberty of gilts. Can. J. A nim. Sci., 67, 553-556.

WALTON J.S., 1984. A note on the effect of exposure to a boar on the development of the reproductive system of immature gilts. Anim. Prod., 39, 483-486.

WALTON J.S., 1985. A note on the effect of exposing gilts to a mature boar at 140 days of age. Anim. Prod., 41, 123 126 